Service social

\title{
L'approche communautaire : un moment pour réfléchir sur l'orientation du service social
}

\section{Marie Drolet}

Volume 35, numéro 3, 1986

Les jeunes et le travail social

URI : https://id.erudit.org/iderudit/706322ar

DOI : https://doi.org/10.7202/706322ar

Aller au sommaire du numéro

Éditeur(s)

École de service social de l'Université Laval

ISSN

1708-1734 (numérique)

Découvrir la revue

Citer ce document

Drolet, M. (1986). L’approche communautaire : un moment pour réfléchir sur l'orientation du service social. Service social, 35(3), 416-444.

https://doi.org/10.7202/706322ar
Résumé de l'article

Marie Drolet nous présente une réflexion sur l'approche communautaire et sur l'importance de sa définition pour le service social et la survie des centres locaux de services communautaires (C.LS.C).

Face aux remises en question de l'État-providence, l'auteure s'interroge sur les dangers de récupération du service social et des groupes autonomes au profit de l'État et de ses besoins économiques. 
DROLET, Marie, travailleuse sociale au C.L.S.C. Les Forges, à Trois-Rivières.

\section{L'approche communautaire : un moment pour réfléchir sur l'orientation du service social *}

\section{Marie Drolet}

Définir l'approche communautaire en des termes précis et clairs apparaît complexe, malgré les faits suivants: ce concept fait partie intégrante du discours dans les centres locaux de services communautaires (C.L.S.C.) ; il est prôné par la Fédération provinciale (F.C.L.S.C.) comme étant son mode particulier d'intervention "originale, novatrice et le plus près possible des communautés " (F.C.L.S.C., 1986: 13); et c'est l'essence même du projet C.L.S.C. (M.A.S., 1983 ; Poupart et al., 1986; Vennat, 1986). Cette vision des problèmes sociaux et de l'action devrait donc être perceptible dans la pratique des C.L.S.C. établis avant 1985; elle devrait être apprise par les intervenants transférés d'autres établissements du réseau des Affaires sociales en 1985.

Le présent article vise à poursuivre la discussion entreprise par Messieurs Denis Bourque et Jean Panet-Raymond dans le numéro de la revue Service Social sur l'organisation communautaire. Notre propos touchera au contenu de ces deux articles; il se veut un autre moment pour réfléchir sur l'approche communautaire en C.L.S.C. et sur son impact possible, soit la récupération des groupes autonomes. Cette discussion nous amènera à dégager les principaux enjeux de la définition actuelle de cette approche. Ces derniers se conjuguent, selon nous, à une redéfinition du service social et à la survie des C.L.S.C.

Précisons que ce texte n'a pas comme objectif de participer à la polémique concernant l'existence des C.L.S.C., tel que le titre la revue L'Actualité de janvier 1987, en posant la question suivante : "A-t-on besoin des C.L.S.C. ? (Demers, 1987.) Nous espérons plutôt, par cette mise en lumière des enjeux concernant à la fois leur existence et leur définition, qu'une discussion de fond autour de la pratique du service social et de la distribution des services sociaux dans notre société soit 
faite par toutes les instances concernées, y compris la population. Nous souhaitons aussi qu'à travers tout ce questionnement ne soient pas oubliés les plus démunis de notre société, éléments importants de la définition du service social et de l'État-providence. Comme le précise Paul-André Comeau, au sujet de tout le débat sur l'État-providence, " au lieu de détourner pudiquement le regard, il vaut mieux engager le débat avant que les dés ne tombent $»$. (Comeau, $1986: 7$. )

\section{Une définition de l’approche communautaire}

Nous choisissons de définir l'approche communautaire à partir de deux textes de base que nous avons retenus en raison de leur publication récente (printemps 1986). Le premier est publié par la Fédération des C.L.S.C. du Québec et le second par le Laboratoire de recherche sur les services communautaires. Nous sommes consciente qu'en raison de notre situation d'intervenante sociale récemment transférée dans un C.L.S.C., la discussion qui suit ne reflétera pas toute la pratique de ces organismes, surtout de ceux qui ont été établis avant 1985. Étant confrontée à l'apprentissage de l'intervention dans un C.L.S.C., ces deux documents ont constitué des références de base; les questions qui ont alors émergé seront ici partagées, en espérant qu'elles seront signifiantes pour certains et complétées par d'autres.

La création d'une culture organisationnelle : le cas des C.L.S.C. est une recherche monographique sur des pratiques de C.L.S.C., menée par le Centre de recherche en gestion de l'Université du Québec à Montréal en collaboration avec la Fédération. Réalisée par Robert Poupart, Jean-Paul Ouellet et Jean-Jacques Simard, l'objectif était de donner des mots à des "pratiques novatrices mal connues et mal reconnues parce qu'elles sont englouties dans les généralisations de l'idéologie " (Poupart et al., 1986 : 13). L'idéologie désigne ici un ensemble de concepts et de représentations qui, dans une organisation, affirme une hiérarchie de valeurs et vise à modeler les comportements individuels et collectifs (Valentish et Gripton, 1982). Poupart et al. (1986) précisent, de plus, qu'une des fonctions de l'idéologie est "d'occulter la réalité " (p. 13) ; leur volonté est donc de donner une représentation juste et scientifique de cette réalité. Leurs observations définiront l'approche communautaire et la personnalité singulière des C.L.S.C. (Poupart et al., 1986). Par cette recherche, la Fédération voulait faire le point sur dix ans de pratique. Elle espère, par la suite, affronter dans une perspective de continuité les diverses difficultés conjoncturelles actuelles: coupures 
budgétaires, essoufflement du personnel et transfert d'intervenants ayant un passé professionnel différent.

Le second texte sur lequel se base notre définition est le Document d'initiation aux types d'intervention communautaire, rédigé par Jérôme Guay et Yolaine Lapointe. Tout d'abord écrit en novembre 1985, à la demande de la conférence des C.L.S.C. de la région 04 (Mauricie/BoisFrancs), il a été republié en 1986. Ce document fait suite à un texte de Jérôme Guay inséré dans l'ouvrage : Psychothérapies : Attention! (1983) sur les aidants non professionnels. L'essence de ce texte a été repris par Hector Ouellet (1984), de la Fédération des C.L.S.C., dans un document de réflexion résumant des éléments bibliographiques sur les services sociaux communautaires. Ce document reprend aussi les principaux éléments du livre de Jérôme Guay, L'intervenant professionnel face à l'aide naturelle (1984).

Les deux textes que nous avons choisis représentent deux perspectives parfois divergentes, parfois complémentaires, qui nous semblent présentes dans le discours de la Fédération et dans les C.L.S.C. Ces positions différentes accroissent la complexité de la définition de l'approche communautaire et créent même des confusions et des ambiguïtés.

\section{Une définition générale : la culture organisationnelle}

La recherche monographique de Poupart et al. (1986) perçoit cette approche, après dix ans de pratique des C.L.S.C., comme le jumelage : du préventif et du curatif ; du social et de la santé ; de l'individuel et du collectif. L'approche communautaire, c'est se soucier de la communauté, lors de la distribution de services et lors de l'intervention visant à produire un changement social. Pour ces auteurs, «c'est d'abord dans la tête [...] elle est autant une attitude et une conception, qu'une méthode de travail " (Poupart et al., 1986: 3).

"La communauté désigne ici ce qui est commun aux membres d'une collectivité particulière; elle repose sur des solidarités ou soudures sociales. Ces solidarités ont deux sources : les valeurs et les intérêts. Ce sont les deux manières pour un être humain de tisser son appartenance à une communauté. " (Poupart et al., 1986 : 64.)

Cette vision permet de définir l'approche communautaire comme une mobilisation de valeurs et comme une utilisation des liens d'intérêt et ce, aux trois niveaux de réseaux présents dans la communauté : 
famille/voisinage, les groupes sociaux, et l'État, qui correspondent aux trois types d'interactions sociales d'un individu.

Tout doit se réaliser dans le respect de cette communauté, de sa culture. La culture désigne "les valeurs, idéaux, croyances, lesquels créent un sentiment d'appartenance collective, inspirent l'action des individus et donnent un sens à l'action " (Poupart et al., 1986: 16). Le respect de la culture " consiste donc à tenir compte de l'inertie du social réalisé, soit le poids de l'histoire, et à l'assumer pour pouvoir la dépasser " (Poupart et al., 1986 : 7).

Par conséquent, l'approche communautaire devient une méthodologie privilégiée pour socialiser un milieu, tout en partageant sa vision du monde (Poupart et al., 1986). Après cet exposé sur " la culture organisationnelle des C.L.S.C. " et sur sa définition de l'approche communautaire, nous pourrions reprendre l'impression de Pierre Vennat (1986) face à ce même document : nous ne savons pas encore de façon précise ce qu'est l'approche communautaire. Elle apparaît pour l'instant comme étant un souci du milieu et une collaboration avec ce dernier, dans tout type d'intervention socio-sanitaire.

Un lien peut être établi entre cette définition et le mémoire présenté en mars 1986 à la Commission d'enquête sur les services de santé et les services sociaux, présidée par le $\mathrm{D}^{r}$ Jean Rochon, où la Fédération des C.L.S.C. poursuit cette orientation en ces termes:

« En tant qu'unités de services de première ligne, les C.L.S.C. devaient dès le départ, dans l'esprit de la réforme, concrétiser les objectifs :

- décentralisation des pouvoirs de décision vers les régions et les usagers ;

- favoriser une étroite participation de ceux-ci à l'expression des besoins du milieu et à l'organisation des soins ;

- promouvoir la prévention et l'action communautaire dans une approche globale et multidisciplinaire ;

- intégrer le volet sanitaire et le volet social dans la distribution des services. " (F.C.L.S.C., 1986 : 9.)

Pour la Fédération, "les C.L.S.C. ont toujours tenté de mettre de l'avant les éléments caractéristiques de leur mission " (F.C.L.S.C., 1986 : 11). En effet, ils ont œuvré pour : l'intégration de la dimension préventive à la dispensation de traitements cliniques, sociaux ou médicaux (niveau d'originalité des pratiques dans les C.L.S.C.) ; et le développement des opportunités de prise en charge, d'entraide et de renforcement du tissu communautaire (autre marque distinctive).

Par ailleurs, la Fédération des C.L.S.C. reprend, dans son mémoire à la Commission Rochon, la définition que donne le Conseil des affaires sociales et de la famille (C.A.S.F.) du concept de santé globale proposé 
par l'Organisation mondiale de la santé (Gaucher et al., 1981). En effet, cette conception désigne un état de bien-être physique et mental où l'on tient compte autant des facteurs sociaux, culturels, économiques que biologiques. L'objectif fondamental devient alors : améliorer l'état de santé et de bien-être des populations ainsi que leur qualité de vie.

"De plus, l'action préventive doit du reste être prioritairement orientée là où elle est susceptible d'avoir le maximum d'impact sur le développement des conditions de vie des populations les plus vulnérables. " (M.A.S., 1983 : 11.)

\section{Une définition plus précise : l'opérationnalisation du service social}

À la différence de la Fédération des C.L.S.C. et de la recherche de Poupart et al. (1986), Jérôme Guay et Yolaine Lapointe (1985) définissent l'approche communautaire "comme étant une nouvelle approche, avec un nouveau cadre conceptuel, de la prise en charge des problèmes de santé physique et mentale" (Guay et Lapointe, 1985). Elle origine de l'essoufflement de l'État-providence (BRIC, 1986; Guay et Lapointe, 1985 ; Ouellet, 1984). Dans le cadre de ce document, la conférence des C.L.S.C. de la région 04 précise que "l'approche communautaire peut être le propre d'un établissement structuré tel un C.L.S.C." (Guay et Lapointe, 1985). Une distinction est alors établie entre cette dernière et l'approche institutionnelle. Pour les auteurs,

"le principal dilemme de notre système socio-sanitaire est le fossé qui existe entre les services publics et la communauté, qui réduit l'efficacité de la prise en charge institutionnelle. En effet, la formation antérieure des intervenant(e)s, leurs valeurs, leur distance face au milieu de vie des personnes font qu'il(elle)s sont appelé(e)s à intervenir lorsque la situation de la personne en besoin est très détériorée et qu'elle commence à être isolée socialement. Il en ressort que la personne étant ainsi plus vulnérable, elle est d'autant plus dépendante de l'intervenant(e). Les chances de réussite de l'intervention s'avèrent plus minces étant donné la faiblesse des ressources de la personne [...]. En conséquence, l'objectif fondamental de l'approche communautaire est donc de combler ce fossé par les niveaux et par les styles d'intervention qui la sous-tendent. " (Guay et Lapointe, 1985.)

En quoi cette définition, dite nouvelle, se différencie-t-elle de l'intention préventive déjà exprimée par la Fédération et de son désir de s'intégrer à la communauté ? Comment peut-on décrire un fossé entre les services publics et la communauté quand la recherche de Poupart et al. (1986) précise que l'action des professionnels de C.L.S.C. 
est déjà de socialiser le milieu tout en respectant sa culture? Est-ce l'expression d'un écart entre l'objectif et la réalité ? Ces questions mettent en évidence la complexité de définir clairement l'approche communautaire : les ambiguïtés et les deux perspectives historiques, où les uns mettent en valeur les dix ans de pratique et les autres, la nouveauté d'une telle approche. Tout ce questionnement met aussi en évidence la difficulté d'intervenir adéquatement dans un milieu, tout en étant intervenant d'une institution, souvent issu d'une autre classe sociale et vivant une autre réalité que celle des clients.

Pour pallier ce fossé entre les institutions et la communauté, les auteurs croient en l'importance de l'aide naturelle: famille, voisins, amis et groupes d'entraide. Ce type d'aide, qui existe déjà dans la communauté, devient ainsi la base de leur action. Ils visent à la susciter, à la mobiliser et à la supporter car elle constitue l'alternative principale à l'intervention professionnelle de type institutionnel. N'y a-t-il pas un risque de sur-utiliser les réseaux d'entraide déjà implantés et de se dégager de la responsabilité de leur apporter une aide directe complémentaire ? La communauté deviendra-t-elle alors un support à l'institution ? L'intervention sociale ne prônait-elle pas auparavant le contraire, soit le support à la communauté par l'intervenant des institutions publiques?

\section{L'approche communautaire : P'intervention dans un quartier}

Guay et Lapointe (1985: 15) réfèrent notamment au modèle du pluralisme participatoire de R. Hadley, du National Institute for Social Work d'Angleterre, dans leur définition de l'approche communautaire. Ce modèle met en évidence la décentralisation et la participation des citoyens, par quartier, pour la distribution de services sociaux. En effet,

"La distribution des services est conçue et réalisée sur la base de petites communautés (quartier, village) et ces petites entités partagent la prise en charge avec le gouvernement central, les bénévoles et les usagers. " (Guay et Lapointe, 1985 : 16.)

Cette conceptualisation basée sur l'aide naturelle et le quartier ne deviendra-t-elle pas une opérationnalisation plus précise de l'approche communautaire déjà définie par Poupart et al. (1986) ? Le quartier ne deviendra-t-il pas ainsi le lieu précis de la socialisation du milieu et l'identification délimitée du terme communauté ? Cette perception de l'intervention sociale qui met en valeur l'aide naturelle et informelle ne redéfinira-t-elle pas l'intervention du professionnel? Dans un quartier, 
il n'aurait qu'à structurer et qu'à supporter les réseaux d'entraide existants, sans véritablement animer ou intervenir pour que se réalise un changement social, comme le proposent Poupart et al. (1986). Le concept de participation locale n'était-il pas déjà mis de l'avant par la F.C.L.S.C. ?

\section{L'approche communautaire : une approche individuelle}

Malgré l'importance accordée à l'intervention de quartier, les auteurs précisent de plus :

"L'apprentissage des types d'intervention communautaire peut se faire dans le cadre de l'intervention individuelle traditionnelle dans le sens que l'on peut partir de la pratique actuelle, telle qu'elle se vit par les praticien(ne)s, pour la modifier et l'élargir en utilisant l'intervention stratégique et l'intervention de support auprès du réseau social. » (Guay et Lapointe, $1985: 57$. )

Peut-on déduire que, si l'intervention de quartier est peu utilisée dans un établissement, l'approche communautaire se réduira à ces deux dimensions de la méthode individuelle? L'institution étatique continuera-t-elle de fournir des services sociaux d'aide individuelle en y ajoutant une intervention stratégique?

En effet, précisons que "l'intervention stratégique ne s'adresse qu'aux problèmes ou parties de problèmes qui sont clairement délimités et dont la solution ne peut se faire indépendamment des facteurs environnementaux" (Guay et Lapointe, 1985: 55). Å quel type de problèmes cette définition réfère-t-elle? L'intervention stratégique vise à faire modifier un comportement ou une situation problématique ; elle se réalise sur une courte durée, sans fournir de support moral au client, qui travaille avec une forte motivation sur son vécu. La première rencontre se fait avec les aidants naturels qui assureront le support moral et l'aide à long terme; cela met en évidence l'importance de l'intervention du professionnel comme support au réseau social. Leur définition de ce type d'intervention se limite aux éléments suivants:

"C'est la seule forme d'intervention qui appartient en propre au niveau professionnel. " (Guay et Lapointe, 1985 : 56.)

"L'intervention stratégique consiste donc essentiellement en l'art de donner des directives aux personnes concernées. [...] L'intervenant(e) aura su maîtriser l'art de manipuler les résistances. " (Guay et Lapointe, 1985 : appendice C2.)

Bref, cette définition de l'approche communautaire désigne trois orientations de l'intervention : tout d'abord le pluralisme participatoire par 
quartier, puis, sinon seulement, le support du réseau naturel et l'intervention stratégique.

\section{L'approche communautaire : la redéfinition du service social}

Comme nous l'exposions précédemment, le premier document n'aurait pas suffi pour avoir une définition claire, précise et opérationnelle de l'approche communautaire. Par ailleurs, dans le second texte, la définition retenue ne met plus en évidence le principe d'alliance avec les marginaux de la société. Ce document n'énonce plus le concept de changement social ni même celui de responsabilité de l'État et de la collectivité face à ses plus démunis, alors que la Fédération les met en valeur dans son mémoire à la Commission Rochon. Cela ne constitueraitil pas une réorientation du service social par rapport à sa définition originale? Ces principes n'étaient peut-être que de l'idéologie, des objectifs et des intentions différentes de la réalité de la pratique sociale (Karsz, 1983), mais ils étaient au moins mentionnés et espérés, alors que dans le texte de Guay et Lapointe (1985) ils n'apparaissent plus.

Élaine Carey-Bélanger, directrice de l'École de service social de l'Université Laval, définit le travail social notamment par la « défense des intérêts des groupes et des populations défavorisés, marginalisés et vulnérables " (Carey-Bélanger, 1985 : 204). Michèle Bourgon, professeure à l'Université du Québec à Montréal, dans un texte publié dans Psychothérapies: Attention! (1983) rappelle que la formation en travail social sert à « favoriser la formation de travailleurs sociaux critiques et équipés dans une perspective de changement social" (Bourgon, 1983a: 148). Gérald Doré définit l'organisation communautaire, ou la méthode collective en service social, comme une pratique sociale qui "consiste à intervenir dans les collectivités, dans le but de susciter leur mobilisation et leur insertion dans des processus de réalisation de changements sociaux» (Doré, 1985: 211). L'approche structurelle, importante en service social individuel, se définit comme une alliance avec les marginaux de la société, où l'environnement est perçu comme source d'oppression ou de support; l'intervenant doit ainsi tenir compte des conditions de vie objectives et matérielles, des conditions de classe et de sexe dans son analyse et dans son action (Bilodeau, 1982 ; Middleman et Goldberg, 1974 ; Moreau, 1982).

N'y a-t-il pas, en effet, danger que ces conceptions du service social soient récupérées pour que cette pratique sociale engendre un retrait de l'État-providence et la reproduction d'une "société néo-libérale »? 
Nous reprenons ici la définition que Diane Lamoureux donne de la récupération lorsqu'elle parle du mouvement social des femmes:

"La récupération commence non pas quand les idées ou les termes [...] sont utilisés mais quand ils le sont pour être "retournés à l'envers", quand ils sont utilisés contre les objectifs du mouvement ou le mouvement lui-même. " (Lamoureux, 1981 : 138.)

Par conséquent, le service social n'est-il pas en train d'être réorienté ? Les pratiques sociales ne dépendent-elles pas d'une perception spécifique du rôle et de la nature de l'État (Ouellet, 1984) ? Ces fonctions de l'État ne sont-elles pas en train de se faire transformer, et la crise de l'Étatprovidence ne touche-t-elle pas le contrat social (Rosanvallon, 1981)? Notre société n'est-elle pas en train de changer, selon la volonté des néo-libéraux et selon les choix économiques devenus critères d'évaluation? N'est-il pas nécessaire à la fois de questionner la pratique du service social préconisée et les choix sociaux qui s'y rattachent? Ces questions entraînent, selon nous, un débat de fond, à réaliser dans les lieux de pratique et dans la société en général. Le présent texte ne constitue qu'une amorce de discussion à poursuivre.

La seconde partie de cet article traitera de la redéfinition de l'Étatprovidence et, par là, du service social, plus spécifiquement dans les C.L.S.C. Elle vise à élargir le débat à sa dimension sociétale, avec une perspective plus historique, globale et structurelle, en espérant qu'un tel type de discussion aura lieu dans d'autres milieux de pratique.

\section{Les enjeux de l'approche communautaire}

II nous apparaissait essentiel de décrire en détail chaque perspective de l'approche communautaire, car chacune deviendra la réponse de la Fédération à des enjeux particuliers. D'une part, la recherche de Poupart et al. (1986), basée sur la réalité vécue dans les C.L.S.C. ainsi que sur le passé et les acquis, soutiendra l'argumentation devant la Commission Rochon, la Commission Parizeau et face au moratoire de la Ministre des services de santé et des services sociaux, Madame Thérèse Lavoie-Roux. D'autre part, le modèle développé par Jérôme Guay, qui peut éventuellement servir de cadre opérationnel, répondra plus spécifiquement à la remise en question de l'État-providence; il deviendra peut-être ainsi l'orientation future des C.L.S.C. 
Enjeux entourant la définition de l'approche communautaire.

\begin{tabular}{|c|c|c|c|c|c|}
\hline Enjeux importants & Acteurs principaux & Idées majeures & Intérêts défendus & Intérêts occultes & Réponse des C.L.S.C. \\
\hline $\begin{array}{l}\text { 1. Moratoire sur le pa- } \\
\text { rachèvement du ré- } \\
\text { seau C.L.S.C., émis } \\
\text { en avril } 1986 \text { (Ga- } \\
\text { gnon, } 1986 \text {; Girard, } \\
\text { 1986a, b et C; Le- } \\
\text { mieux, 1986; Presse } \\
\text { canadienne,1986a } \\
\text { et b; Sainte-Marie, } \\
\text { 1986a) }\end{array}$ & Ministre Lavoie-Roux & $\begin{array}{l}\text {-Pas de C.L.S.C. créé } \\
\text { en } 1986 \text {; pas decharte } \\
\text { remise aux dix-huit } \\
\text { C.L.S.C. en attente } \\
\text {-C.L.S.C. mis sur pied } \\
\text { au fur et à mesure que } \\
\text { les ressources finan- } \\
\text { cières seraient dispo- } \\
\text { nibles } \\
\text {-Un comité de travail } \\
\text { sous la responsabilité } \\
\text { du Dr Jacques Brunet } \\
\text { étudiela rentabilité des } \\
\text { C.L.S.C. }\end{array}$ & $\begin{array}{l}\text {-Régler le problème du } \\
\text { désengorgement des } \\
\text { hôpitaux } \\
\text {-Pas de C.L.S.C. en mi- } \\
\text { lieu urbain, surtout en } \\
\text { raison d'autres res- } \\
\text { sources dans la com- } \\
\text { munauté } \\
\text {-Favoriser la santé avant } \\
\text { lesservices sociaux (Gi- } \\
\text { rard, 1986a) } \\
\text {-Abolir certains pro- } \\
\text { grammes pour garantir } \\
\text { l'accessibilité et l'uni- } \\
\text { versalité des services }\end{array}$ & $\begin{array}{l}\text {-Défendre le budget de } \\
\text { son Ministère au Con- } \\
\text { seil des ministres et de- } \\
\text { vant le Conseil du Tré- } \\
\text { sor } \\
\text {-Résister à des pressions } \\
\text { comme le Rapport sur } \\
\text { la privatisation et la dé- } \\
\text { réglementation publié } \\
\text { parM. Paul Gobeil,pré- } \\
\text { sident du Conseil du } \\
\text { Trésor en juillet } 1986\end{array}$ & $\begin{array}{l}\text {-Un C.L.S.C. est une } \\
\text { solution économique } \\
\text { parla prévention qui } \\
\text { favorise la bonne san- } \\
\text { té de la population } \\
\text {-Capacité accrue de } \\
\text { prendre de multiples } \\
\text { mandats ministériels } \\
\text { vu l'approche nou- } \\
\text { - velle des intervenants } \\
\text { qui réduit la tâche } \\
\text { pour chaque client } \\
\text { (rentabilitéaccrue par } \\
\text { la quantité) } \\
\text { - Mise en valeur de la } \\
\text { définition de santé } \\
\text { globaleoù segreffent } \\
\text { les services sociaux }\end{array}$ \\
\hline
\end{tabular}




\section{Enjeux entourant la définition de l'approche communautaire.}

\begin{tabular}{|c|c|c|c|c|c|}
\hline Enjeux importants & Acteurs principaux & Idées majeures & Intérêts défendus & Intérêts occultes & Réponse des C.L.S.C. \\
\hline & $\begin{array}{l}\text { Association de méde- } \\
\text { cins }\end{array}$ & $\begin{array}{l}\text {-À la Commission Ro- } \\
\text { chon,l'Association des } \\
\text { conseils de médecins, } \\
\text { dentistes et pharma- } \\
\text { ciens du Québec de- } \\
\text { mande de redéfinir les } \\
\text { C.L.S.C. qui sont une } \\
\text { porte d'entrée à "cer- } \\
\text { tains socialistes doc- } \\
\text { trinaires et qui consti- } \\
\text { tuent un réseau paral- } \\
\text { lèle démédicalisé à la } \\
\text { médecine qui est pra- } \\
\text { tiquée en cabinets pri- } \\
\text { vés et en polyclini- } \\
\text { ques" (Gagnon, 1986) }\end{array}$ & $\begin{array}{l}\text {-Une qualité de la mé- } \\
\text { decine basée sur la spé- } \\
\text { cialisation et les tech- } \\
\text { nologies de pointe } \\
\text { pour assurer de meil- } \\
\text { leurs services àla clien- } \\
\text { tèle et pour prolonger } \\
\text { l'espérance de vie }\end{array}$ & $\begin{array}{l}\text {-La défense de la mé- } \\
\text { decine payée à l'acte } \\
\text { qui assure un revenu } \\
\text { plus substantiel et } \\
\text { contrôlable surtout } \\
\text { pour les spécialistes } \\
\text {-Assurer le libre marché } \\
\text { des cabinets privés et } \\
\text { des polycliniques sur- } \\
\text { tout en milieu urbain } \\
\text {-Aucun contrôle étati- } \\
\text { que sur la profession }\end{array}$ & $\begin{array}{l}\text {-Économie pour la } \\
\text { Régie d'assurance- } \\
\text { maladie parlesalariat } \\
\text { des médecinsaulieu } \\
\text { du paiement à l'acte } \\
\text {-Médecine plus pré- } \\
\text { ventive, plusglobale } \\
\text { avec moins d'abus à } \\
\text { cause du salariat }\end{array}$ \\
\hline & $\begin{array}{l}\text { Association des direc- } \\
\text { tions de la protection } \\
\text { de la jeunesse (D.P.J.) }\end{array}$ & $\begin{array}{l}\text {-Des services doivent } \\
\text { être fournis aux enfants } \\
\text { en besoin de protec- } \\
\text { tion } \\
\text {-Redéfinir le choix des } \\
\text { clientèles cibles }\end{array}$ & $\begin{array}{l}\text {-Désengorger la liste d'at- } \\
\text { tente de chaque Centre } \\
\text { de services sociaux } \\
\text { (C.S.S.) } \\
\text {-Ne pas avoir à trop } \\
\text { limiterl'entrée des dos- } \\
\text { siers }\end{array}$ & $\begin{array}{l}\text {-Compenser le manque } \\
\text { depersonnel en C.S.S. } \\
\text { dû aux transferts d'in- } \\
\text { tervenants en C.L.S.C. } \\
\text { en } 1985 \\
\text {-Considérer les services } \\
\text { deprévention comme } \\
\text { étant plus du luxe de- } \\
\text { vant la nécessité deser- } \\
\text { vices curatifs dans un } \\
\text { contexte de coupures } \\
\text { budgétaires }\end{array}$ & $\begin{array}{l}\text {-D'une part, refuser } \\
\text { les dossiers D.P.J. } \\
\text { pourmaintenirla dis- } \\
\text { ponibilité des inter- } \\
\text { venants à faire de la } \\
\text { prévention, et ainsi } \\
\text { ne pas modifier leur } \\
\text { mission } \\
\text {-D'autre part, négocier } \\
\text { certaines responsa- } \\
\text { bilités liées à la pro- } \\
\text { tection sociale afin } \\
\text { d'avoir les budgets } \\
\text { connexes et ainsi as- } \\
\text { surer leur survie }\end{array}$ \\
\hline
\end{tabular}




\begin{tabular}{|c|c|c|c|c|c|}
\hline $\begin{array}{l}\text { 2. Désengorgement } \\
\text { desurgences d'hô- } \\
\text { pitaux (F.C.L.S.C., } \\
1986 ; \text { Presse cana- } \\
\text { dienne, 1986; Sa- } \\
\text { vary, 1986a et b) }\end{array}$ & Ministre Lavoie-Roux & $\begin{array}{l}\text {-Deux clientèles prin- } \\
\text { cipales utilisent abusi- } \\
\text { vement et non adé- } \\
\text { quatement les ur- } \\
\text { gences des hôpitaux : } \\
\text { les personnesâgéeset } \\
\text { les personnes avec des } \\
\text { troubles psychiatriques } \\
\text { ou autres problèmes } \\
\text { de santé mentale } \\
\text { Mesures préconisées : } \\
\text { 1. services intensifs à } \\
\text { domicile, surtout } \\
\text { pour personnes } \\
\text { âgées áes } \\
\text { 2. équipes gériatriques } \\
\text { avec services psy- } \\
\text { chogériatriques de } \\
\text { visites à domicile } \\
\text { 3. quelquesplacesen } \\
\text { centre d'accueil } \\
\text { 4. lits de soins de } \\
\text { courte durée } \\
\text { 5. centre de crise en } \\
\text { santé mentale }\end{array}$ & $\begin{array}{l}\text {-Maintenir les per- } \\
\text { sonnes âgées à domi- } \\
\text { cile, dans leur milieu, } \\
\text { pour une qualité de } \\
\text { vieet une vieillessese- } \\
\text { reine } \\
\text {-Maintenir les per- } \\
\text { sonnes qui ont des trou- } \\
\text { blesmentauxdansleur } \\
\text { milieu, pour assurer } \\
\text { leur qualité de vie et } \\
\text { leur fonctionnement } \\
\text { dans la société }\end{array}$ & $\begin{array}{l}\text {-Ce maintien à domicile } \\
\text { coûte moins cher que } \\
\text { toute autre forme d'hé- } \\
\text { bergement } \\
\text {-Diminuer l'entrée de } \\
\text { personnes âgées dans } \\
\text { le réseau des affaires } \\
\text { sociales en bloquant } \\
\text { l'entrée par les hôpi- } \\
\text { taux } \\
\text {-Avoir des budgets pour } \\
\text { des servicesà domicile } \\
\text { actuellement peu adé- } \\
\text { quats, surtout avecl'aug- } \\
\text { mentation de l'espé- } \\
\text { rance de vie } \\
\text {-Peu de questionne- } \\
\text { mentsur les causes so- } \\
\text { ciales des maladies men- } \\
\text { tales ou des troubles } \\
\text { de fonctionnement }\end{array}$ & $\begin{array}{l}\text {-Les C.L.S.C. ont déjà } \\
\text { lesoucides deux be-- } \\
\text { soins majeurs en san- } \\
\text { té identifiés par le } \\
\text { C.A.S.F., soit : vieil- } \\
\text { lissement accéléré de } \\
\text { lapopulation et pro- } \\
\text { blèmes croissants de } \\
\text { maladie mentale } \\
\text {-Les C.L.S.C. ont } \\
\text { conscience des pro- } \\
\text { blèmes de notre so-- } \\
\text { ciété : vieillissement } \\
\text { de lapopulation avec } \\
\text { augmentation del'es- } \\
\text { pérance de vie mais } \\
\text { avec aussi des épi- } \\
\text { sodes plus fréquents } \\
\text { d'incapacitélimitant } \\
\text { l'autonomie ; de- } \\
\text { mande accrue de ser- } \\
\text { vices à domicile ; li- } \\
\text { mites financières de } \\
\text { l'État ; problèmes } \\
\text { croissants d'adapta- } \\
\text { tionsocialeet de san- } \\
\text { té mentale dus à no-- } \\
\text { tre société plus diffi- } \\
\text { cile } \\
\text {-Services à domicile } \\
\text { déjà structurés avec } \\
\text { des services sociaux } \\
\text { complémentaires aux } \\
\text { services de santé }\end{array}$ \\
\hline
\end{tabular}


Enjeux entourant la définition de l’approche communautaire.

\begin{tabular}{|c|c|c|c|c|c|}
\hline Enjeux importants & Acteurs principaux & Idées majeures & Intérêts défendus & Intérêts occultes & Réponse des C.L.S.C. \\
\hline & $\begin{array}{l}\text { Association des hôpi- } \\
\text { taux }\end{array}$ & $\begin{array}{l}\text {-Rééquilibrer leur bud- } \\
\text { get, pour ne pas avoir } \\
\text { de déficit qui n'est plus } \\
\text { épongé par le Minis- } \\
\text { tère } \\
\text {-Avoir des budgets sup- } \\
\text { plémentaires pour la } \\
\text { technologie médicale } \\
\text { et l'immobilisation }\end{array}$ & $\begin{array}{l}\text { - La qualité des soins aux } \\
\text { patients } \\
\text {-La réduction du temps } \\
\text { d'attente pour des ser- } \\
\text { vices }\end{array}$ & $\begin{array}{l}\text {-Avoir les budgets pour } \\
\text { des équipes géria- } \\
\text { triques, centre decrise } \\
\text { en maladie mentale, } \\
\text { même une partie du } \\
\text { budget pour services } \\
\text { à domicile et familles } \\
\text { d'accueil } \\
\text {-Assurerlepouvoir mé- } \\
\text { dical sur tous les ser- } \\
\text { vices,dont lesservices } \\
\text { sociaux }\end{array}$ & $\begin{array}{l}\text {-Avoir déjà la défini- } \\
\text { tion desantéglobale } \\
\text {-Recevoir les budgets } \\
\text { pour les servicesétant } \\
\text { donné l'infrastructure } \\
\text { prête à assurer les } \\
\text { services,etpourévi- } \\
\text { terle dédoublement } \\
\text { des services } \\
\text {-Éviterl'établissement } \\
\text { de services privés à } \\
\text { domicile ; assurerl'u- } \\
\text { niversalité de services } \\
\text { à domicile; assurer } \\
\text { l'universalité etlagra- } \\
\text { tuité (ou presque) des } \\
\text { services } \\
\text {-S'associer avec des } \\
\text { organismesà but non } \\
\text { lucratif pour distri- } \\
\text { bution de services } \\
\text { aveccontrat à forfait }\end{array}$ \\
\hline $\begin{array}{l}\text { 3. Commission Ro- } \\
\text { chon (Commission } \\
\text { d'enquête sur les } \\
\text { services de santé } \\
\text { et les services so- } \\
\text { ciaux) }\end{array}$ & $\begin{array}{l}\text { Secteur privé (médecins, } \\
\text { gensd'affaires, psycho- } \\
\text { logues ou autres bu- } \\
\text { reaux privés de relation } \\
\text { d'aide) }\end{array}$ & -Laisser le libre marché & $\begin{array}{l}\text {-Instauration d'un sec- } \\
\text { teur privé de distribu- } \\
\text { tion de soins pour ser- } \\
\text { vices à domicile, hé- } \\
\text { bergement de per- } \\
\text { sonnes âgées, même }\end{array}$ & $\begin{array}{l}\text {-Réduire l'universalité } \\
\text { et l'accessibilité desser- } \\
\text { vices pourbaisserl'im- } \\
\text { pôt et pour réduire } \\
\text { aussil'ingérence del'É- } \\
\text { tat }\end{array}$ & $\begin{array}{l}\text {-Promouvoir les acquis } \\
\text { face à l'universalité } \\
\text { des services } \\
\text {-Défendre la position } \\
\text { des plus démunis et } \\
\text { l'intervention au ni- }\end{array}$ \\
\hline
\end{tabular}


Discuter de la situation actuelleseIon trois paramètres: le vieillissement de la population, la technologie de pointe et le changement de mentalités (Bélanger, 1986a ; Doré etFortin, $1986 ; \mathrm{Gi}$ rard, 1986; Guay, 1984 ; Panet-Raymond, 1986 ; Sainte-Marie, 1986b)

\begin{tabular}{|c|c|c|c|c|}
\hline & & $\begin{array}{l}\text { pour des services mé- } \\
\text { dicaux et psychoso- } \\
\text { ciaux privés }\end{array}$ & -S'assurer des profits & $\begin{array}{l}\text { veau des secteurs à } \\
\text { risques }\end{array}$ \\
\hline $\begin{array}{l}\text { Organismes communau- } \\
\text { taires }\end{array}$ & $\begin{array}{l}\text {-Reconnaissance de leur } \\
\text { action et de leur survie } \\
\text { financière } \\
\text {-Assurer leur autonomie } \\
\text { d'action, notamment } \\
\text { face au contrôle étati- } \\
\text { que, par la gestion du } \\
\text { financement } \\
\text {-Prôner la débureau- } \\
\text { cratisation, la dépro- } \\
\text { fessionnalisation }\end{array}$ & $\begin{array}{l}\text {-Assurer des services de } \\
\text { qualité à toute la po- } \\
\text { pulation selon sa réalité } \\
\text { et ses besoins } \\
\text {-S'assurer que les déci- } \\
\text { sions ne soient pas } \\
\text { prises par la Ministre } \\
\text { avant le dépôt du rap- } \\
\text { port Rochon, ce qui } \\
\text { viendrait structurer et } \\
\text { légitimer la privatisation } \\
\text { et la fermeture du ré- } \\
\text { seau des affaires so- } \\
\text { ciales }\end{array}$ & $\begin{array}{l}\text {-Assurer leur survie, leur } \\
\text { financement et lesem- } \\
\text { plois des permanents } \\
\text {-Défendre des acquis } \\
\text { de l'État-Providence } \\
\text {-Promouvoir un chan- } \\
\text { gement social en faveur } \\
\text { de la classe populaire } \\
\text { et en faveur de la re- } \\
\text { prise de la place des } \\
\text { femmes dans une so- } \\
\text { ciété plus égalitaire }\end{array}$ & $\begin{array}{l}\text {-Eviterla privatisation, } \\
\text { par la famille, l'ani- } \\
\text { mation de quartier } \\
\text { et les ressources com- } \\
\text { munautaires } \\
\text {-Promouvoirla parti- } \\
\text { cipation déjà établie } \\
\text { des instances locales } \\
\text { dans la gestion des } \\
\text { soins des C.L.S.C. } \\
\text {-Maximiserl'approche } \\
\text { co mm m n a u ta ire } \\
\text { comme une interven- } \\
\text { tion de collaboration } \\
\text { et desocialisation du } \\
\text { milieu ; travailler au } \\
\text { niveau des quartiers, } \\
\text { avec les ressources } \\
\text { d'aide naturelle et les } \\
\text { groupes existants }\end{array}$ \\
\hline
\end{tabular}


Enjeux entourant la définition de l'approche communautaire.

\begin{tabular}{|c|c|c|c|c|c|}
\hline Enjeux importants & Acteurs principaux & Idées majeures & Intérêts défendus & Intérêts occultes & Réponse des C.L.S.C. \\
\hline $\begin{array}{l}\text { 4. Commission Pari- } \\
\text { zeau } \\
\text { Commission, sous } \\
\text { la présidence de Jac- } \\
\text { ques Parizeau, sur } \\
\text { le rôle des munici- } \\
\text { palités et organisée } \\
\text { parl'Union des mu- } \\
\text { nicipalités du Qué- } \\
\text { bec (Bélanger, } \\
\text { 1986b) }\end{array}$ & $\begin{array}{l}\text { Commission Parizeau et } \\
\text { municipalités }\end{array}$ & $\begin{array}{l}\text {-Hypothèse que certains } \\
\text { services sociaux et ser- } \\
\text { vices de maintien à do- } \\
\text { micile soient transférés } \\
\text { aux municipalités }\end{array}$ & $\begin{array}{l}\text {-Éviter le dédoublement } \\
\text { des instances locales } \\
\text { de décision }\end{array}$ & $\begin{array}{l}\text {-Avoir le contrôle et le } \\
\text { budget concernant ces } \\
\text { services, surtout si les } \\
\text { C.L.S.C. maximisent } \\
\text { leurgestion et leuror- } \\
\text { ganisation de services } \\
\text { par quartier }\end{array}$ & $\begin{array}{l}\text {-Réaffirmer son pou- } \\
\text { voir local et la vérita- } \\
\text { ble participation des } \\
\text { instances locales dans } \\
\text { la direction des ser- } \\
\text { vices } \\
\text {-Maximiserl'associa- } \\
\text { tion C.L.S.C.-muni-i- } \\
\text { palités ou C.L.S.C.- } \\
\text { union des municipa- } \\
\text { lités régionales de } \\
\text { comté, pouruneap- } \\
\text { proche globale et } \\
\text { communautaire de } \\
\text { la santé }\end{array}$ \\
\hline
\end{tabular}


Ce tableau ne se veut pas un exposé exhaustif de tous les enjeux entourant l'existence et la définition des C.L.S.C.; il ne veut pas y présenter tous les acteurs en présence, leurs idées et leurs intérêts. II identifie plutôt, de façon schématique, les multiples discours et les diverses pressions concernant la survie des C.L.S.C. et, par conséquent, de l'approche communautaire et du service social. Cela nous aidera à mieux comprendre la réalité sociale qui entoure ce débat spécifique et à supporter une discussion plus générale sur les choix de notre société en matière de services sociaux, fournis par les divers établissements du réseau étatique et par les groupes autonomes.

Le point de vue de la Fédération, qui a été décrit surtout par la culture organisationnelle, est perceptible dans les réponses des C.L.S.C. à ses différents opposants. L'approche communautaire, l'intervention " originale, novatrice et la plus près possible des communautés » (F.C.L.S.C., 1986: 13), développée sur dix ans, légitimise l'existence même des C.L.S.C.

Le jumelage du préventif et du curatif, un niveau d'originalité des pratiques des C.L.S.C., met en évidence l'importance de la prévention pour éviter les coûts élevés des soins. Tenter d'améliorer le niveau de santé et de bien-être de la population, promouvoir des changements de mentalités, percevoir les problèmes individuels selon une vision globale deviennent donc des moyens pour prévenir une augmentation des coûts des soins médicaux et sociaux. Même si les facteurs environnementaux incluent les conditions économiques, de sexe et de classe sociale, et même si la Fédération met en valeur la prévention auprès des plus démunis, nous ne considérons pas que cette approche permette une analyse en profondeur des causes structurelles des conditions économiques, objectives et matérielles qui défavorisent certaines classes de la société.

Le discours sur la participation locale, y incluant le conseil d'administration, valide l'intégration du C.L.S.C. dans la communauté. L'intervention auprès des réseaux d'aide naturelle, le pluralisme participatoire, le travail de quartier, la collaboration avec les organismes communautaires, la socialisation dans le respect du milieu viennent confirmer cette implantation dans la localité. À la Commission Parizeau, les municipalités auront donc à tenir compte des C.L.S.C. véritablement implantés dans leur communauté.

Cette position apporte un argument pour le parachèvement du réseau de C.L.S.C. Vu, d'une part, la nécessité et la demande des communautés locales pour avoir un C.L.S.C., et vu, d'autre part, l'effet positif, pour la F.C.L.S.C., de la présence d'un tel organisme dans les localités, la Ministre y verra ainsi un investissement important et rentable. 
Le C.L.S.C. devient donc un moyen adéquat pour " apaiser " les insatisfactions de la population que l'approche communautaire canalisera en fonction du maintien de la "paix sociale". Le C.L.S.C. devient aussi l'organisme privilégié pour la distribution des services de soins à domicile et de santé mentale, auxquels la F.C.L.S.C. a déjà réfléchi (F.C.L.S.C., 1986). Il désengorgera, en effet, les hôpitaux et la Direction de la protection de la jeunesse ; il aura ainsi des budgets supplémentaires connexes en structurant, dans les quartiers, des services d'entraide ou des organismes bénévoles pour maintenir les personnes à domicile. Cette organisation collective à peu de frais compensera, par conséquent, I'hébergement étatique à haut frais et évitera la privatisation des soins de santé (du moins pour les personnes qui n'auront pas un revenu suffisant pour se payer des soins privés).

L'approche communautaire qui vise notamment à diminuer l'aide directe auprès du client (Guay et Lapointe, 1985) ne devient-elle pas un moyen efficace et économiquement rentable d'alléger la tâche des intervenants sociaux, victimes de coupures de postes et qui subissent un allourdissement de leur tâche dû à l'augmentation des mandats que doivent accepter les C.L.S.C. pour prouver leur rentabilité et assurer leur survie? L'intervention individuelle ne devient-elle pas ainsi un moyen rentable pour renforcer la position des établissements dans l'organisation des quartiers ? Les administrateurs ne seront-ils pas aussi portés à individualiser l'échec de la résolution des problèmes individuels et sociaux? Pour eux, les facteurs d'échecs peuvent alors être : I'intervenant qui ne réussit pas à maîtriser les résistances, le client et son milieu qui sont responsables de ne pas se prendre en charge. Quelle sera la place accordée au rôle du travailleur social s'il fait moins d'interventions d'aide directe auprès de la clientèle, en individuel et en collectif ? Offrira-t-il des services de support ou des services sociaux réduits?

Ces derniers propos peuvent paraître corporatistes ; mais ne s'agitil pas là d'une redéfinition de la pratique du service social ? Quelle sera la teneur de l'animation de quartier? L'aide naturelle sera-t-elle surutilisée au «service de l'État " ? Ou encore, ce quartier ne pourrait-il pas devenir un lieu de conscientisation, vu la concrétisation, dans sa réalité, de la dimension collective et sociétale des problèmes individuels et sociaux? Autant de questions, autant d'inconnues. Ne se dessine-t-il pas ici, à travers des enjeux pour la survie d'un établissement, une nouvelle pratique sociale et, indirectement, une redéfinition de l'État et de la société?

Bref, l'enjeu de l'existence et de la survie des C.L.S.C. est de taille, celle de la définition du service social aussi. Nous espérons qu'un tel questionnement sur la pratique d'une profession, mais aussi sur sa 
valeur sociale, soit poursuivi par d'autres intervenants issus d'autres lieux de pratique, car la remise en question de l'État-Providence nous affecte tous et toutes.

\section{Remise en question de l'État-providence}

\section{Un choix économique de société : l'État-providence}

La "nouvelle» approche communautaire, définie par Guay et Lapointe (1985), est issue de l'essoufflement de l'État-providence. Pour insérer cette pratique dans une perspective sociétale, et pour comprendre les enjeux plus globaux de l'intervention en C.L.S.C., il faut tout d'abord définir l'État-providence afin d'établir un cadre plus précis à la discussion ultérieure sur sa remise en question et sur les impacts qui en découlent pour l'intervention sociale. Cette perspective plus historique permet, selon nous, d'élargir un débat professionnel à un débat de société. La définition que nous avons retenue s'inspire de l'approche en C.L.S.C. et ne reflète donc pas tout le contexte socio-économique et politique qu'il recouvre. L'État-providence désigne donc, pour nous, l'intervention massive de l'État (au Québec, à partir des années 1960) dans la gestion des conditions économiques favorables au développement de la société ; la perspective sociale supporte cet essor économique.

"L'État-Providence était la solution miracle aux déficiences réelles ou appréhendées du système de marché [...] de nouvelles sociétés d'État dans plusieurs secteurs remplaçaient les entreprises privées diagnostiquées comme défaillantes [...] le gouvernement québécois représentait l'instrument par lequel on espérait accroître le rôle, alors marginal, des francophones dans l'économie [...] le poids relatif de l'ensemble des administrations publiques dans l'économie s'est mis à augmenter rapidement [...] : paiement de transfert aux particuliers, subventions aux entreprises et achats de biens et services. " (Bélanger et Lamonde, 1986 : 111.)

Nous voulons rappeler les notions économiques sous-jacentes à cette définition car les critiques actuelles, face à l'État, occultent qu'il s'agissait, d'une part, d'un choix de société et que, d'autre part, ce choix, maintenant considéré comme défavorable à la collectivité et à l'économie, a déjà été perçu comme moteur de l'économie.

À cette définition économique de l'État-providence, faite par Bélanger et Lamonde (1986), se rattache une perspective sociale qui influe plus directement sur la définition de l'approche communautaire. 
L'État intervient pour réduire les inégalités entre les classes sociales, par des paiements de transfert aux particuliers, mais aussi par des services sociaux, médicaux et scolaires universels et accessibles à tous. Recevoir une aide de la collectivité n'est plus perçu comme une charité mais comme un droit, une justice pour tous; cela devient un moyen pour atteindre l'égalité des chances pour tous les citoyens (Vaillancourt, 1986). L'État symbolise la cohésion sociale, les droits collectifs et la socialisation universelle des biens publics (Brunelle, 1986; Comeau, 1986 ; Ouellet, 1984 ; Rosanvallon, 1981 ; Vaillancourt, 1986). Il est alors une conscience de la collectivité et il a une conscience sociale. C'est dans cette perspective que fut publié le rapport Castonguay-Nepveu, que fut votée la Loi 65 , que furent établis les C.L.S.C. et que fut dessinée la première ébauche de l'approche communautaire.

\section{Une remise en question économique de l'État-providence : justification de la survie des C.L.S.C.}

Vu notre souci d'accorder une perspective sociétale à notre réflexion sur le service social, nous présenterons une remise en question plus générale de l'État-providence, puis nous aborderons celle des C.L.S.C.

Quatre problèmes fondamentaux sont maintenant généralement associés à l'État-providence :

- la trop grande part de consommation privée et publique, au détriment des investissements;

- l'expansion de l'économie clandestine, c'est-à-dire le travail au noir où la main-d'œuvre est utilisée dans des emplois précaires aux conditions minimales de travail ;

- les responsabilités de l'État, qui se sont étendues au rythme de l'apparition des problèmes économiques ;

- l'inefficacité des paiements de transfert aux particuliers pour réduire les inégalités sociales (Lesourne, 1981, dans : Bélanger et Lamonde, 1986).

Des éléments socio-politiques s'ajoutent aussi à ces problèmes économiques. "Le gonflement des bureaucraties appartient pour une partie à une logique interne" (Bélanger et Lamonde, 1986; Brunelle, 1986 : 6 ; F.C.L.S.C., 1986). Une autre caractéristique importante de la critique actuelle contre l'État-providence est l'accord des néo-libéraux et des mouvements sociaux pour interroger le côté extensif de l'État, c'est-à-dire le contrôle social qui en découle et la bureaucratie qui s'en nourrit. Cette critique est formulée très différemment par l'un et l'autre groupe. Les néo-libéraux prônent le libre marché, la privatisation des 
entreprises d'État et le retour à des valeurs de croissance économique et à des responsabilités individuelles; mais surtout, ils veulent la réduction des coûts et des demandes à l'État afin de diminuer les impôts des particuliers et des entreprises ainsi que le déficit étatique (Bélanger et Lamonde, 1986; Friedman, 1983 ; Lemieux, 1986 ; Rosanvallon, 1981). Les mouvements sociaux cherchent, pour leur part, à conserver, dans leurs luttes pour une équité collective, leur autonomie face au contrôle étatique, notamment par le contrôle des subventions (Hamel, 1983; Panet-Raymond, 1985).

À ces problèmes socio-économiques associés à l'État-providence se greffe une critique économique des C.L.S.C. et donc, indirectement, de l'approche communautaire et du service social.

Dans leur analyse de l'état-providence, L'utopie du plein emploi, Jean-Pierre Bélanger et Pierre Lamonde formulent très clairement que les services de santé et sociaux coûtent trop cher et qu'il s'agit d'un point névralgique de diminution des dépenses de l'État. "Les coûts de santé progressent vite et en bonne partie indépendamment de la croissance de l'économie et des recettes publiques." (Bélanger et Lamonde, 1986 : 123.) Pour eux, quatre causes principales influencent cette montée des coûts. De cette énumération nous pouvons en déduire que les C.L.S.C. seront une solution économiquement rentable. Nous y retrouvons, en effet, le discours de la F.C.L.S.C. face aux enjeux concernant sa survie. Il s'agit de :

- la valeur sociale accordée aux soins et à la santé (les C.L.S.C. répondront à ce souci d'avoir une bonne santé en prônant la prévention) ;

- le vieillissement de la population (le maintien à domicile fourni par les C.L.S.C. répondra à cette généralisation des besoins et des demandes d'aide inhérents au vieillissement) ;

- P'inadaptation des services de santé, notamment en gériatrie et en santé mentale (la notion de santé globale déjà développée dans les C.L.S.C. les rend immédiatement prêts à faire face à cette clientèle présente, entre autres, dans les urgences des hôpitaux et à la D.P.J.) ;

- la haute technologie médicale et le paiement à l'acte (le salariat des médecins), qui facilite le développement du concept de prévention et le contrôle étatique de la pratique médicale, s'avèrent, selon eux, déjà efficaces dans les C.L.S.C.

Ces quatre causes de la montée des coûts de santé associées aux difficultés de financement (Girard, 1986) et aux pressions privées renforcent l'importance, déjà mentionnée, de l'économie dans les choix actuels de pratique des C.L.S.C. 


\section{La récupération des C.L.S.C. de la critique de l'État-providence : justification de la « nouvelle» approche communautaire}

Pour poursuivre cette discussion sur le questionnement de l'Étatprovidence et sur l'impact de l'économique dans les choix des pratiques des C.L.S.C., nous présenterons les concepts sous-jacents à l'origine de l'approche communautaire prônée par Guay et Lapointe (1985). Elle vient, pour eux, de la position financière difficile de l'État qui affecte notamment les C.L.S.C. par des coupures budgétaires. C'est ainsi que, dans un souci de rationalisation, d'efficacité et de rentabilité, elle prend toute sa place, notamment : I'incitation à la participation accrue de la population dans la distribution même des services et la réduction de la définition de la tâche de l'intervenant social.

Par ailleurs, la critique de l'omniprésence de l'État et du contrôle qui y est sous-jacent est reprise par plusieurs auteurs (Guay et Lapointe, 1985 : 2-3 ; Rosanvallon, 1981; Ouellet, 1983a et b ; Grand'maison, 1984 ; Ouellet, 1984 ; Bourque, 1985 ; Panet-Raymond, 1985 ; F.C.L.S.C., 1986; De Ravinel, 1987).

L'État-providence a-t-il vraiment déresponsabilisé le citoyen au point qu'il soit devenu dépendant des services publics? Les C.L.S.C., jusqu'à présent, n'ont-ils pas travaillé à favoriser «le développement de l'entraide et de l'esprit communautaire " (F.C.L.S.C., 1986) ? Ce discours n'occulte-t-il pas une "gestion de la décroissance" économique de notre société (Duchastel, 1983 ; F.C.L.S.C., 1986 ; Mercier, 1983 ; Ouellet, 1983b) ? En effet, dans un contexte de restrictions budgétaires , " comment gérer la décroissance sans porter atteinte à la qualité, [à la quantité], à l'accessibilité [et à l'universalité] des services? " (F.C.L.S.C., 1986: 8) ?

Ce dilemme s'avère encore plus difficile parce qu'une situation de crise économique comme celle des années 1980 a accru les difficultés sociales dues, notamment, au chômage (Boisjoli et Lafontaine, 1983; Duchastel, 1983; Maheu, 1983; Tardy, 1983). Ce contexte accroît donc les demandes d'aide alors que les recettes publiques et les ressources collectives diminuent. Par ailleurs, le tissu social n'est plus celui du Québec des années précédant la Révolution tranquille, où la famille élargie assumait tous ses membres. De plus, les maladies mentales s'accroissent avec le stress de la société postindustrielle (Lamontagne et al., 1985).

C'est ainsi que ce discours, qui réutilise les critiques du contrôle étatique, devient pour nous une justification idéologique pour affronter les difficultés économiques, soit : les diminutions des recettes de l'État, le déficit étatique, la volonté de ne pas augmenter les impôts, et la 
volonté de réduire les services de l'État. Quand les propos de la F.C.L.S.C. sont comparés à ceux de Brian Mulroney, cité ci-après, nous considérons que l'approche communautaire, telle que prônée par Guay et Lapointe (1985), a vraiment une fonction idéologique qui occulte le retrait de l'État, même si les valeurs de solidarité sociale sont, selon nous, valables, et même si les études sur les réseaux sociaux prouvent leur importance pour les individus (Bouchard, 1983 ; Corin et al., 1984). Le tout est ici récupéré, c'est-à-dire utilisé à d'autres fins que celles de leur définition première. En effet, "le bénévolat représente aujourd'hui la méthode la plus rentable de fonctionnement au Canada " (Mulroney, 1984, dans : Panet-Raymond, $1985: 342$ ). Il est ainsi associé à la rentabilité économique et non à la conscience sociale et à la responsabilité collective face à ses membres. Le réseau des bénévoles est conscient de cette récupération possible (Boulet, 1986). Une étude sur les réseaux sociaux des personnes âgées met en garde contre sa surutilisation (Corin et al., 1984). La discussion sur les causes sociales de l'accroissement de la demande d'aide est donc, selon nous, éclipsée ; le seul argument qui est actuellement entendu demeure celui des coûts élevés des services sociaux, où l'intervenant doit faire une "utilisation adéquate " des " réseaux sociaux tissés d'entraide " (De Ranivel, 1987). II y a alors, pour nous, un véritable danger d'utiliser à outrance les réseaux d'entraide déjà existants, de ne plus leur apporter assez de support et de moins offrir d'aide directe nécessaire et ce, pour des raisons économiques.

Par ailleurs, les classes les plus privilégiées de notre société utiliserontelles les services bénévoles lors d'un besoin d'aide, ou ne paieront-elles pas des services privés? Les classes les plus démunies, celles qui sont identifiées comme les plus vulnérables, spécialement en période de crise, auront-elles un autre choix que celui de s'entraider bénévolement ? N'est-ce pas là aussi un moyen de récupérer le chômage et la maind'œuvre de l'économie clandestine, de redonner aux femmes leurs rôles traditionnels de support familial et aux familles leurs responsabilités comme base de la société ? Où est l'analyse scientifique de la réalitté sociale actuelle qui guiderait une intervention sociale adéquate et adaptée aux années 1980 ?

\section{La récupération des groupes autonomes}

Aux deux précédentes positions sur la critique de l'État-providence s'en ajoute une troisième: la première prône que les C.L.S.C. sont rentables, la deuxième justifie la " nouvelle " approche communautaire, alors que la troisième récupère les groupes autonomes (collaborateurs 
et partenaires des services étatiques, notamment des C.L.S.C. dans la distribution de services sociaux).

Les trois solutions proposées par Pierre Rosanvallon à la Crise de l'État-providence (1981) s'appliquent directement à l'approche communautaire et aux C.L.S.C. (Ouellet, 1984). Ces trois solutions concernent :

- la socialisation plus souple des organismes publics par la débureaucratisation et la rationalisation des services (les C.L.S.C. vus comme les plus petites unités du système actuel des services de santé et des services sociaux, deviennent, pour la F.C.L.S.C., les organismes à mettre en valeur) ;

- la décentralisation de l'État vers les collectivités locales qui verront leurs responsabilités et leurs tâches accroître (la participation du pouvoir local et des quartiers dans les C.L.S.C. devient un argument justifiant leur survie) ;

- l'autonomisation ou le transfert à des collectivités non publiques des tâches de service public ; ceci est, pour Rosanvallon, la voie la plus innovatrice de la transformation de l'État-providence et de son retrait (c'est la définition même, selon nous, de l'approche communautaire de Guay et Lapointe, 1985).

Pour Pierre Rosanvallon (1981), il faut que ces solutions s'inscrivent dans un plan d'ensemble où l'État doit, tout d'abord, réduire les demandes. Les C.L.S.C., en privilégiant, comme critère de réception d'aide, la responsabilité individuelle et la motivation/capacité de la clientèle à s'assumer, réduisent ainsi les demandes de services aux clients volontaires. Les C.L.S.C. ne deviendront-ils pas une "porte de fermeture", plutôt qu'une "porte d'entrée", comme organismes de première ligne du système de services de santé et de services sociaux ? L'approche communautaire ne devient-elle pas une justification à ne plus fournir d'aide directe à certaines clientèles particulières en les renvoyant à leur responsabilité personnelle? Rosanvallon met aussi en valeur la production d'une plus grande visibilité des résultats de l'utilisation des fonds publics dans la communauté. Le pluralisme participatoire et la participation locale à la distribution de services ne deviennent-ils pas un moyen pour l'État, et pour les C.L.S.C., d'être vus et connus ? Finalement, il prône un "réencadrement de la solidarité dans la société " (Rosanvallon, 1981 : 112), ce qui signifie, pour lui, récupérer pour l'État :

— l'existence des réseaux souterrains de solidarité familiale ;

- les mouvements autonomes d'entraide (Lavoie, 1983) avec leur dynamisme et leur recherche d'autonomie et d'identification ;

- les mouvements sociaux qui, par leur militantisme, luttent pour un changement vers une équité sociale et qui surtout, pour lui, 
créent des services sociaux alternatifs (Lamoureux, 1981 ; Melucci, 1983 ; Panet-Raymond, 1985 ; Touraine, dans : Rocher, 1969).

Cela représente, en termes clairs, le fondement même de l'approche définie par Guay et Lapointe (1985) et endossée par certains textes de la Fédération des C.L.S.C. (C.L.S.C.-04, dans: Guay et Lapointe, 1985; Ouellet, 1983a ; Ouellet, 1984). Est-ce que ce concept de "solidarité" tient véritablement compte de tous les changements de notre société cités précédemment, soit : l'éclatement de la famille élargie, l'entrée des femmes sur le marché du travail, la solitude et l'isolement dans les villes? N'y a-t-il donc pas un autre danger de récupération, soit celui des groupes autonomes et alternatifs de services au profit de l'État et en fonction de ses besoins économiques (Bourque, 1985 : Doré et Fortin, 1985 ; Panet-Raymond, 1985) ? Les groupes ont besoin d'une liberté de pensée et d'action pour vivre et se développer. Que leur arrivera-t-il dans un tel contexte?

Par ailleurs, Pierre Rosanvallon met en valeur le modèle anglais d'entraide adopté depuis 1977 (Rosanvallon, 1981: 128-129), qui est repris par Jérôme Guay, soit le modèle du pluralisme participatoire de R. Hadley, d'Angleterre (Guay et Lapointe, 1985 : 3). De même, Hector Ouellet relie l'approche communautaire au rapport Barclay (Ouellet, 1983a ; Ouellet, 1984), portant sur la refonte des services sociaux et du rôle du travailleur social en Angleterre (Barclay, 1982; Bourque, 1985; Ouellet, 1983a; Ouellet, 1984). Sera-t-elle, au Québec, la nouvelle définition de la communauté qui remplacera celle énoncée par Poupart et al. (1986) ?

Et voilà, le service social serait redéfini, réorienté, récupéré. L'essence de la définition de cette profession donnée précédemment (l'aide aux plus démunis et le changement social) est "retournée à l'envers"; les usagers, la population et les groupes aideront l'État, par le biais de l'approche communautaire, à assumer son déficit et ses difficultés à offrir des services sociaux, alors que les travailleurs sociaux pourront devenir des agents d'information, et de mise en application. Pour qui voulons-nous œuvrer : pour l'État ou pour les plus démunis de notre société ? Cette question met en évidence, une fois de plus, la complexité inhérente à l'intervention sociale, mais elle mérite tout de même qu'on se la pose dans les C.L.S.C. et dans d'autres milieux de service social.

\section{Conclusion}

En guise de conclusion, il faut replacer cette réflexion, qui peut paraître comme étant les "désillusions d'une agente de changement ", 
dans une perspective plus sociétale. La conjoncture actuelle donne à la profession de service social l'occasion de se questionner, de se réévaluer et se réajuster à la réalité des années 1980, telle qu'elle est vécue dans les C.L.S.C., dans les Centres de services sociaux et dans tout autre lieu de pratique sociale.

Mais un tel bouillonnement, présent à l'intérieur et à l'extérieur des délibérations de la Commission Rochon, peut aussi donner lieu à des discussions parfois stériles et destructrices entre des individus, des professions et des établissements. Nous espérons que le débat de fond autour de la redéfinition actuelle de notre société, notamment à travers le rôle de l'État, aura lieu et qu'il aura lieu avec ceux qui sont concernés : les usagers et la population. Nous souhaitons aussi que les plus démunis de notre société ne seront pas oubliés, eux qui sont l'une des raisons d'être du service social et de l'État-providence. La société québécoise a obtenu que les services sociaux soient un droit et non une charité ; il ne faudrait pas que cela devienne le privilège des plus nantis. Les principes du service social constituaient peut-être une idéologie qui occultait une fonction de maintien de la "paix sociale" (Karsz, 1983), mais ils avaient le mérite d'être mentionnés et de viser le changement social et l'établissement de rapports sociaux plus égalitaires.

En terminant cette réflexion sur le service social, il ne faudrait pas oublier les groupes autonomes, un milieu d'intervention complémentaire aux services étatiques : " se priver de cette avant-garde, c'est condamner les services officiels à la sclérose » (Cliche, 1986b : 4).

\section{Note}

* L'auteur tient à souligner que ce texte a été écrit avant le dépôt du Rapport du comité de réflexions et d'analyse des services dispensés par les C.L.S.C., présidé parle Dr J. Brunet, Québec, 10 mars 1987, 92 pages.

\section{Références bibliographiques}

Barclay, Peter, Social Workers, Their Role and Tasks: the Report of a Working Party Set Up in October 1980 at the Request of the Secretary of State for Social Services, Londres, National Institute for Social Work, Bedford Square Press, 1982.

BÉlANGer, Jean-Pierre, "Notre mémoire : le C.L.S.C. de demain... au centre des services sociaux", Commission Rochon, Fédé Express, vol. 9, no 8, 1986a : 9-11. 
BélANGER, Pierre, "Notre mémoire: le C.L.S.C. de demain, véritable instance locale ", Commission Parizeau, Fédé Express, vol. 9, no 8, 1986b : 12-13.

Bélanger, Pierre et Pierre Lamonde, L'utopie du plein emploi: croissanceéconomique et aspiration au travail, Québec 1971-2001, Montréal, Boréal Express, 1986.

BILODEAU, Guy, "L'approche à orientation structurelle", document de travail inséré dans le : Recueil de textes "Service Social auprès de la famille ", SVS14994, Québec, Université Laval, hiver 1982: 3-20.

BoISJOL, Johanne et Danielle LAfONTAINE, "Mutation des sphères publique et privée : l'arrivée aux limites d'un régime", Les enjeux de la décroissance; actes du Colloque de l'Association canadienne des sociologues et des anthropologues de langue française (1982), Montréal, Albert Saint-Martin, 1983 : 149-173.

BouCHARD, Camil, "Prétendre prévenir ", Revue internationale d'action communautaire, 11/51, $1984:$ :21-29.

BOULET, Pierre, "Fini l'État-providence... le gouvernement investit dans le bénévolat "; "des millions qui valent des milliards"; " histoire d'une volte face pour le moins révélatrice"; "éviter le piège de la privation déguisée ", Le Soleil, 12 avril 1986 : B1.

Bourgon, Michèle, "Dépayser l'individuel ", Psychothérapies : Attention !, Sillery, Québec Science, Presses de l'Université du Québec, 1983 : 143-147.

BOURQUE, Denis, "L'approche communautaire en centre local de services communautaires : les enjeux en cause et les conditions requises ", Service Social, vol. 34 , nos $2-3,1985: 328-340$.

Brunelle, Dorval, "L'économie narcissique de Pierre Lemieux", chroniqueréplique, Le Devoir, 17 juillet $1986: 7$.

Bureau de recherche et d'intervention communautaire (BRIC), Programme de consultation/formation en intervention communautaire, Québec, BRIC, 1986.

Carey-Bélanger, Élaine, "Éditorial: la Commission Rochon: enjeux pour le service social, les services sociaux et la société ", Service Social, vol. 34, nos 23, 1985 : 202-206.

CLICHE, Vincent, "La vie en noir ", chronique bloc-notes, Le Soleil, 30 septembre 1986 : B4.

COMEAU, Paul-André, "Le débat sur le néo-libéralisme", chronique bloc-notes, Le Devoir, 17 juillet 1986 : 7.

CORIN, Ellen et al., "Entre les services professionnels et les réseaux sociaux : les stratégies d'existence des personnes âgées ", Sociologie et sociétés, vol. XVI, no 2, 1984 : 89-105.

Demers, Dominique, "A-t-on besoin des C.L.S.C. ?", L'Actualité, vol. 12, no 1, janvier 1987: 40-44.

DE RAVINel, Hubert, "Quelques propositions face à l'impasse des soins et des services sociaux ", La Presse, 10 janvier 1987 : D3.

DORE, Gérald, "L'organisation communautaire : définition et paradigme ", Service Social, vol. 34, nos 2-3, 1985 : 210-231. 
DORÉ, Gérald et Denis FORTIN, «Avant-propos : l'organisation communautaire à contre-courant de l'État-Business ", Service Social, vol. 34, nos 2-3, 1985 : 206-210.

DUCHASTEL, Jules, "Les enjeux sociaux d'une problématique de la décroissance", Les enjeux de la décroissance; actes du Colloque de l'Association canadienne des sociologues et des anthropologues de langue française (1982), Montréal, Albert Saint-Martin, 1983 : 15-23.

Fédération des C.L.S.C. du Québec (F.C.L.S.C.Q.), Les C.L.S.C. et la réforme socio-sanitaire au Québec: pour un bilan prospectif. Point de vue de la F.C.L.S.C.Q. sur la situation du système québécois de la santé et des services sociaux, présenté à la Commission Rochon, Montréal, F.C.L.S.C.Q., 1986.

Friedman, Milton et Rose Friedman, La tyrannie du statu quo, préface d'Alain Cotta, traduit de l'américain par Patrice Hoffman, Paris, Éditions JeanClaude Lattès, 1984, 264p.

GAGNON, Damien, "La ministre Lavoie-Roux n'est pas contente de leur travail ; une épée de Damoclès sur les C.L.S.C. ", Le Soleil, 10 mai 1986a : B3.

GaGnon, Damien, "Cri d'alarme lancé au nom des jeunes", Le Soleil, 3 juin 1986b : A6.

GAGNON, Damien, "Craignant d'avoir une image négative, les C.L.S.C. espèrent que l'État précisera enfin ses attentes à leur sujet ", Le Soleil, 23 octobre 1986c: A15.

GaUCher, Dominique, France Laurendeau et Louise-Hélène Trottier, "Parler de la vie: l'apport des femmes à la sociologie de la santé ", Sociologie et sociétés, vol. XIII, no 2, 1981: 139-153.

GIRARD, Maurice, série d'articles sur la Commission Rochon, Le Soleil :

1. "La santé : pas plus chère ici qu'ailleurs", 18 août 1986 : A2.

2. "Privatisation et rémunération ont animé les grands débats", 19 août 1986 : A2.

3. "Santé : l'épineuse question du financement", 20 août 1986: A24.

4. "Les femmes et les personnes âgées, principaux clients des services de santé" ", 21 août 1986 : A13.

5. "Rochon est prudent face au gouvernement ", 22 août 1986: C13.

Grand'Maison, Jacques, Le C.L.S.C. : en position privilégiée pour une stratégie de milieu, Conférence donnée au Congrès de la Fédération des C.L.S.C. du Québec, Québec, octobre 1984.

GUAY, Jérôme, "Les aidants non professionnels", Psychothérapies: Attention! Sillery, Québec science, Presses de l'Université du Québec, 1983 : 157-181.

GuAY, Jérôme, L'intervenant professionnel face à l'aide naturelle, Chicoutimi, Gaëtan Morin, 1984.

GUAY, Jérôme et Yolaine LAPOINTE, Document d'initiation aux types d'intervention communautaire, Québec, Laboratoire de recherche sur les services communautaires de l'Université Laval, 1985.

HAMEL, Pierre, "Crise de la redistribution étatique et financement des organisations populaires", Revue internationale d'action communautaire, 10/50, 1983 : 63-77.

KarSz, Saul, "Pour une analyse scientifique du Travail Social», Cahiers de pratiques sociales, nos 2-3, mai-juin-juillet $1983: 5-25$. 
LAMONTAGne, Yves, Francine LAVOIE et Lise TeSSIER, La santé mentale : prévenir, traiter et réadapter efficacement, Avis du Comité de la santé mentale au Québec, Québec, Gouvernement du Québec, 1985.

Lamoureux, Diane, "Mouvement social et luttes des femmes", Sociologie et sociétés, vol. XIII, no 2, 1981 : 131-139.

LAVoIE, Francine, "Les groupes d'entraide", Psychothérapies; Attention ! Sillery, Québec science, Presses de l'Université du Québec, 1983 : 181-203.

LEMIEUx, Pierre, série d'articles sur "Y a-t-il un gouvernement libéral au Québec ? ", Le Devoir:

1. "La privatisation: un gouvernement rempli de bonnes intentions", 12 juillet 1986, A1,8.

2. "Le trésor public et la tyrannie du statu quo ", 14 juillet 1986, A1,6.

3. "Déréglementation: la nécessité d'une philosophie", 15 juillet 1986, A1,8.

4. "Les droits de l'homme : libéralement un gouvernement sans gouvernail ", 16 juillet 1986, A1,8.

MAHEU, Louis, "Crise sociale, mouvements sociaux et pratiques de changement social ", Crise économique, transformations politiques et changements idéologiques; Actes du Colloque de la société québécoise de science politique (1983), Montréal, Association canadienne-française pour l'avancement des sciences, 1983 : 477-497.

MeluCCI, Alberto, "Mouvements sociaux, mouvements post-politiques ", Revue internationale d'action communautaire, 10/50, 1983: 13-31.

MERCIER, Jean, "Les effets des compressions budgétaires sur l'accès et le développement des services", Les enjeux sociaux de la décroissance; actes du Colloque de l'Association canadienne des sociologues et des anthropologues de langue française (1982), Montréal, Albert Saint-Martin, 1983 : 69-83.

Middleman, R.R. et G. Goldberg, Social Service Delivery, a Structural Approach to Social Work Practice, New York, Columbia University Press, 1974.

Ministère des affaires sociales (M.A.S.), Le partage des responsabilités C.S.S.C.L.S.C. en matière des services sociaux, Québec, M.A.S., 1983.

MOREAU, Maurice, "L'approche structurelle familiale en service social : le résultat d'un itinéraire critique ", Revue internationale d'action communautaire,7/ 47, 1982 : 159171.

OUELlet, Hector, Les services sociaux au Québec. Éléments de réflexion pour les années 1980: 1re partie et $2^{\mathrm{e}}$ partie sur les enjeux actuels, Montréal, F.C.L.S.C.Q., 1983a.

OUELLET, Hector, « Les conséquences des compressions budgétaires sur l'accès et le développement des services ", Les enjeux de la décroissance; actes du colloque de l'Association canadienne des sociologues et des anthropologues de langue française (1982), Montréal, Albert Saint-Martin, 1983b : 83-91.

OUELlet, Hector, Les services sociaux communautaires : quelques éléments bibliographiques, Montréal, F.C.L.S.C.Q., 1984.

PANET-RAYMOND, Jean, "Nouvelles pratiques des organisations populaires... Du militantisme au bénévolat au service de l'État ", Service Social, vol. 34, nos 23, $1985: 340-353$. 
Poupart, Robert, Jean-Paul Ouellet et Jean-Jacques Simard, La création d'une culture organisationnelle: le cas des C.L.S.C., Montréal, F.C.L.S.C.Q. et Centre de recherche en gestion de l'Université du Québec à Montréal, 1986.

Presse canadienne, "Thérèse Lavoie-Roux opte pour la consolidation du réseau existant ; pas d'autres C.L.S.C. », Le Nouvelliste, 25 avril 1986a, p. 3.

Presse canadienne, "Pas de décision sur le réseau des C.L.S.C. avant d'avoir le rapport Brunet ", Le Soleil, 20 octobre 1986b : B8.

ROCHER, Guy, Introduction à la sociologie générale, tomes 1-3, Montréal, H.M.H., 1969, ("Regard sur la réalité sociale».)

Rosanvallon, Pierre, La crise de l'État-providence, (édition revue, corrigée et mise à jour), Paris, Seuil, 1981, 190p.

SAINTE-MARIE, Anne, "Un moratoire décourageant pour les dix-huit équipes de secteurs ; le parachèvement du réseau ", Fédé Express, vol. 9, no 8, 1986a : 6-8.

SAINTE-Marie, Anne, "Les hypothèses de solution proposées par les C.L.S.C."; Commission Rochon, Fédé Express, vol. 9, no 7, 1986b : 3-5.

SAVARY, Claude, "Engorgement des urgences; les C.L.S.C. seraient la solution", Le Nouvelliste, 23 juillet 1986, p. 5.

SavarY, Claude, "Compressions dans les hôpitaux; la réponse du M.S.S.Q. connue à la mi-août ", Le Nouvelliste, 25 juillet 1986b, p. 3.

TARDIf, Évelyne, "Le mouvement des femmes et la crise", Crise économique, transformations politiques et changements idéologiques; actes du colloque de la société québécoise de science politique (1983), Montréal, Association canadienne-française pour l'avancement des sciences, 1983 : 353-373.

VAILlANCOURT, Yves, série d'articles sur "l'État et le social au Québec ", Le Devoir : 1. "À l'époque où l'État québécois jouait un rôle supplétif", 2 août 1986, $A 1,8$.

2. "Le retour du pendule : l'attrait du recours à la privatisation ", 4 août $1986, \mathrm{~A} 1,6$.

3. "Le modèle ontarien, vu de plus près ", 5 août $1986, A 1,8$.

4. "Un véritable dossier test : celui de l'aide sociale", 6 août 1986, A1,8.

VALENTISH, Mary et J. GRIPTON, "Ideological perspectives on sexual assault of women : Complication for social work ", conférence donnée au Congrès de l'Association canadienne des écoles de service social, Ottawa, 1982.

Vennat, Pierre, "L'expérience unique des C.L.S.C. ", La Presse, 23 août 1986 : B2. 